\title{
Rapid intraoperative method for the identification of metastatic lymph nodes from thyroid carcinoma
}

\author{
longshun shi ${ }^{1}$, Minhao Xie ${ }^{1}$, Guo $\mathrm{Wu}^{2}$, Jun Fan ${ }^{1}$, Mingming Guo ${ }^{1}$, Runlin Yang ${ }^{1}$, Jue \\ Zhang $^{3}$, Yi Zhang ${ }^{3}$, Bin Zhou ${ }^{3}$, and Zhongwei $\mathrm{Lv}^{4}$ \\ ${ }^{1}$ NHC Key Laboratory of Nuclear Medicine, Jiangsu Key Laboratory of Molecular Nuclear \\ Medicine \\ ${ }^{2}$ Hubei University of Medicine Affiliated Dongfeng Hospital \\ ${ }^{3}$ Jiangsu Institution of Nuclear Medicine \\ ${ }^{4}$ Shanghai 10th People's Hospital, Tongji University School of Medicine, Shanghai, China
}

April 28, 2020

\begin{abstract}
In this study, we established a rapid method for intraoperative identification of thyroid carcinoma metastatic lymph nodes. We developed a rapid method of thyroid globulin $(\mathrm{Tg})$ detection called $\mathrm{Tg}$-POCT based on time-resolved fluorescence immunoassay and validated reagent performance. Thyroid and lymph node tissues with metastatic thyroid cancer were considered as the positive group, and muscle, fiber, fat, and other tissues were considered as the negative control group to verify the above method combined with fine needle puncture. CV of the same batch number was $8.38 \%$ and that of different batch numbers was $11.24 \%$. The minimum detection limit was $0.02 \mathrm{ng} / \mathrm{mL}$. The test conformity of tissue samples was $100 \%$ by using the self-designed reference value. Thus, Tg-POCT is feasible as a rapid intraoperative method to identify thyroid cancer metastatic lymph nodes.
\end{abstract}

Rapid intraoperative method for the identification of metastatic lymph nodes from thyroid
carcinoma Longshun Shi ${ }^{\text {ad }}$, Minhao Xie ${ }^{\text {ad }}$ (Co-first authors), Guo $\mathrm{Wu}^{\mathrm{b}}$, Jun Fan ${ }^{\text {ad }}$, Mingming Guo ${ }^{\text {ad }}$, Runlin Yang ${ }^{\text {ad }}$, Jue Zhang ${ }^{\text {ad }}$, Yi Zhang ${ }^{\text {ad }}$, Bin Zhou ${ }^{\text {ad }}$, Zhongwei Lv ${ }^{c}$ (Co-corresponding authors)

In this study, we established a rapid method for intraoperative identification of thyroid carcinoma metastatic lymph nodes. We developed a rapid method of thyroid globulin ( $\mathrm{Tg}$ ) detection called Tg-POCT based on time-resolved fluorescence immunoassay and validated reagent performance. Thyroid and lymph node tissues with metastatic thyroid cancer were considered as the positive group, and muscle, fiber, fat, and other tissues were considered as the negative control group to verify the above method combined with fine needle puncture. CV of the same batch number was $8.38 \%$ and that of different batch numbers was $11.24 \%$. The minimum detection limit was $0.02 \mathrm{ng} / \mathrm{mL}$. The test conformity of tissue samples was $100 \%$ by using the self-designed reference value. Thus, Tg-POCT is feasible as a rapid intraoperative method to identify thyroid cancer metastatic lymph nodes.

Introduction

In recent years, we attempted to determine thyroglobulin ( $\mathrm{Tg}$ ) content by fine needle aspiration (FNA) eluent, which showed positive significance in the qualitative analysis of thyroid tumor-related cervical lymph nodes [1-2]. Tg is a macromolecule glycoprotein, synthesized only by thyroid epithelial cells, with a relative molecular weight of $660 \mathrm{KD}$, and its presence can indicate the existence of thyroid follicular epithelial cells. 
In addition to the high levels content of $\mathrm{Tg}$ in normal thyroid tissues, high levels of $\mathrm{Tg}$ are observed in welldifferentiated thyroid cancer tissues and lymph node tissues with thyroid cancer metastasis [3]. Therefore, $\mathrm{Tg}$ detection by puncture cell eluent can be used as a supplement to FNA to improve accuracy in identifying the metastatic lymph nodes of thyroid cancer. In addition, the rapid detection of $\mathrm{Tg}$ in puncture cell eluent during surgery will significantly improve the quality of thyroid cancer surgery and reduce the risk of postoperative recurrence and metastasis.

In this study, a rapid detection method based on time-resolved fluorescence microsphere immunochromatographic assay of $\mathrm{Tg}$ microspheres was established; we also verified its performance and conducted preliminary clinical comparisons.

1. NHC Key Laboratory of Nuclear Medicine, Jiangsu Key Laboratory of Molecular Nuclear Medicine,Jiangsu Institute of Nuclear Medicine. Wuxi, Jiangsu, China 214063.

2. Department of Nuclear Medicine, DongFeng Affiliated Hospital of Hubei University of Medicine. Shiyan, Hubei, China

3. Department of Nuclear Medicine, Shanghai 10th People's Hospital, Tongji University School of Medicine, Shanghai, China

4. . Department of Radiopharmaceuticals, School of Pharmacy, Nanjing Medical University, Nanjing 211166, China

+ Footnotes relating to the title and/or authors should appear here.

Electronic Supplementary Information (ESI) available: [details of any supplementary information available should be included here]. See DOI: 10.1039/x0xx00000x

Materials and Methods

Data

A pair of antibodies (From Sigma-Aldrich Company, Building C, qiantan world trade center (phase ii), no. 3, lane 227, Dongyu road, Pudong new area, Shanghai, China) was used to capture the Tg present in samples. Goat anti-rabbit immunoglobulin and rabbit IgG (From Sigma-Aldrich Company, Building C, qiantan world trade center (phase ii), no. 3, lane 227, Dongyu road, Pudong new area, Shanghai, China) were used as quality control to ensure the accuracy of test results. Thyroglobulin antigen (From Roche Group, 1100 Longdong Road, Pudong new area, Shanghai, China) was used to substitute tissue in the experiments. Fluorescent microspheres (From Thermo Fisher Scientific, 27 Xinjinqiao road, Pudong new area, Shanghai, China) bind to antibodies and were used for detecting antigen-antibody binding. Analyzing membrane, water absorbing paper, sample pad, and binding pad (From GE,1 Yongchang North Road, Beijing, China) were included in the testing system. The test samples were obtained from the pathological examination of tissue types confirmed by the pathology department. Thyroid and lymph node tissues with metastatic thyroid cancer were included in the positive group, and muscle, fiber, fat, and other tissues were included in the negative control group.

Methods

After ultrasonic resuspension, $1 \mathrm{~mL}$ of the fluorescent microspheres were placed in a $1.5 \mathrm{ml} \mathrm{EP}$ tube and centrifuged at room temperature for $15 \mathrm{~min}$ at a centrifugation speed of $1 \times 104 \mathrm{r} / \mathrm{min}$. Further, $0.02 \mathrm{~mol} / \mathrm{L}$ 2-(N-morph) ethanesulfonic acid(MES) buffer was used. The $\mathrm{pH}$ was washed from 2.0-8.0 twice and the 1(3-Dimethylaminopropyl)-3-ethylcarbodiimide hydrochloride (EDC) activation solution containing $2 \mathrm{mg} / \mathrm{mL}$ was added. After being activated at room temperature in darkness for $30 \mathrm{~min}$, washing with $0.02 \mathrm{~mol} / \mathrm{L}$ phosphate buffer (PBS), ultra-pure water, $0.05 \mathrm{~mol} / \mathrm{L}$ HEPES buffer or $0.05 \mathrm{~mol} / \mathrm{L}$ Tris-HCl buffer was performed twice. The above liquid was added according to the microsphere: the mass of the antibody was 1-20 $\mu \mathrm{g}$, and the labelled antibody was added and conjugated for $2 \mathrm{~h}$ in darkness. After the binding was completed, a sealant equivalent to $1 / 20$ of the volume of the coupling solution was added. The sealant was $0.02 \mathrm{~mol} / \mathrm{L}$ PBS buffer containing $10 \%$ bovine serum albumin (BSA), ultra-pure water, $0.05 \mathrm{~mol} / \mathrm{L}$ HEPES buffer or $0.05 \mathrm{~mol} / \mathrm{L}$ Tris- $\mathrm{HCl}$ buffer. The buffer system of the sealant was the same as that of the binding 
buffer system. The reaction was allowed to proceed for $30 \mathrm{~min}$ in darkness. After centrifugation for 15 min, the supernatant was discarded and washed twice with deionized water. Then, the supernatant was redissolved with $0.02 \mathrm{~mol} / \mathrm{L}$ PBS buffer containing 1\% BSA (pH 7.4), and the above liquid was diluted to $1 \%-10 \%$ of the original concentration using $0.02 \mathrm{~mol} / \mathrm{LPBS}$ buffer containing $1 \%$ BSA and $0.1 \%$ polysorbate and then stored as sample reaction solution at 4.(Figure1. Labeling of fluorescent microspheres)

NC membranes were laid on the bottom plate of polyvinyl chloride (PVC), and the coated antibody and quality control antibody were diluted to $0.1-1 \mathrm{mg} / \mathrm{mL}$ using $0.02 \mathrm{~mol} / \mathrm{L}$ PBS solution $(\mathrm{pH} 7.4$ ) containing $1 \%$ sucrose. The two were evenly sprayed on the NC film at an interval of $0.5 \mathrm{~cm}$ and $1.25 \mathrm{~L} / \mathrm{cm}$ to form a detection line $(\mathrm{T})$ and quality control line $(\mathrm{C})$. The diluent was sprayed on the binding pad with a quantity of $150 \mu \mathrm{L}$ per $1.5 \times 30 \mathrm{~cm}^{2}$. After $4 \mathrm{~h}$ of drying at 30 in the drying box, we laid absorbent paper and sample pads on both sides of the NC film. At $35 \%$ humidity, the test strip was cut into $0.4 \mathrm{~cm}$-wide strips at 25 and put into the clamp to form the test strip.( Figure2. The composition of speed measuring card

)

The above reagent allowed to warm to room temperature. Tg antigen (approximately $100 \mathrm{ng} / \mathrm{mL}$ ) was considered to be a high-value sample, and normal saline was considered to be a zero sample. Further, 50 $\mu \mathrm{L}$ of $\mathrm{Tg}$ antigen was added to the reagent strip and $50 \mu \mathrm{L}$ of sample reaction solution was added. The ratio of high-value sample/zero sample was defined as signal-to-noise ratio, and the method with the highest signal-to-noise ratio was selected as the optimal scheme.(Figure3. Testing Flow)

Statistical analyses

SPSS 19.0 software was used to analyze various performance indices of Tg-POCT kits and to assess the specificity and sensitivity of clinical tissue samples.

Results

During the research and development process, we found that $\mathrm{pH}$ and the amount of antibody had a great impact on the quality of the reagent, so we assessed the effect of the main indicators of each key link in the production process.

Effect of $\mathrm{pH}$ of activated liquid

The $\mathrm{pH}$ gradient of the microsphere during activation was $2.0-8.0$, and the optimum $\mathrm{pH}$ was 4.5.(Figure4. effect of $\mathrm{pH}$ on activation)

Effect of the $\mathrm{pH}$ of the coupling liquid

The $\mathrm{pH}$ gradient of the microsphere in the conjugated antibody was 5.0-12.0, and the optimum $\mathrm{pH}$ was 8.0.(Figure5. effect of $\mathrm{pH}$ on coupling)

Effect of buffer solution

During microsphere coupling, the buffer systems were PBS buffer, ultrapure water, HEPES buffer, and tris- $\mathrm{HCl}$ buffer. PBS was the optimum buffer solution.(Figure6.effect of buffer solution)

Effect of amount of antibody for labelling

The amount of antibody used for labelling was $1-20 \mu \mathrm{g}$. The optimum amount of antibody was $10 \mu \mathrm{g}$. ( Figure7. effect of antibody labelled amount)

Effect of amount of antibody for coating

The amount of antibody used for coating was $1-20 \mu \mathrm{g} ; 0.8 \mu \mathrm{g}$ was the optimum amount of antibody for coating.( Figure8.effect of antibody coated amount)

Effect of dilution ratio of fluorescent microspheres 
The dilution ratio gradient of fluorescent microspheres was $1 \%-10 \%$, and the optimum dilution ratio was $3 \%$.( Figure9.effect of dilution ratio of fluorescent microsphere)

Performance verification

\section{Standard curve}

Tg reference calibrators of $10 \mathrm{ng} / \mathrm{mL}, 50 \mathrm{ng} / \mathrm{mL}, 100 \mathrm{ng} / \mathrm{mL}, 200 \mathrm{ng} / \mathrm{mL}$, and $300 \mathrm{ng} / \mathrm{mL}$ were added to Tg-POCT test paper as a drop of $60 \mu \mathrm{L}$. After reaction at 30 for $5 \mathrm{~min}$, the test was performed. The T/C value, which was the signal value, was measured five times and $\mathrm{CV}$ was counted. According to the statistical method, $\mathrm{T} / \mathrm{C}$ ratio of the detected sample was taken as the ordinate, and the concentration of $\mathrm{Tg}$ standard was taken as the $\mathrm{X}$-coordinate. The equation was established, and the standard curve was synthesized. Linear regression coefficient was 0.976 . The high value was partly due to the hook effect. Overall, the curve was linear and smooth.( Table1. standard curve Figure10. standard curve)

Table1. standard curve

\begin{tabular}{lll}
\hline reference calibrators $(\mathrm{ng} / \mathrm{mL})$ & signal value & $\mathrm{CVs}(\%)$ \\
10 & $1.31 \pm 0.054$ & 4.12 \\
50 & $2.79 \pm 0.119$ & 4.27 \\
100 & $4.08 \pm 0.162$ & 3.97 \\
200 & $5.69 \pm 0.205$ & 3.60 \\
300 & $6.81 \pm 0.273$ & 4.01 \\
\hline
\end{tabular}

\section{Stability and degree of precision}

The $60 \mu \mathrm{L}$ Tg reference standards $(10 \mathrm{ng} / \mathrm{mL}, 300 \mathrm{ng} / \mathrm{mL})$ were added to the test strip, and the fluorescence values of the quality control line and detection line were read on the immunoassay machine after reaction at 30 for $5 \mathrm{~min}$, and the measurements were repeated 10 times. The reagent was placed at 4 for 6 months and tested again. The test was repeated 10 times. The CVs in $10 \mathrm{ng} / \mathrm{mL}$ point test batch were $9.62 \%$, $13.05 \%, 7.14 \%$, and $9.43 \%$ in $300 \mathrm{ng} / \mathrm{mL}$. Considering that the fluctuation of fluorescence values in the low concentrations were higher than those in the high concentrations owing to human error and other reasons, it was normal that the low concentration value $\mathrm{CV}$ was higher than the high concentration $\mathrm{CV}$ value. In conclusion, the Tg-POCT reagent has good stability and degree of precision.( Table2. stability and degree of precision)

Table2. stability and degree of precision

\begin{tabular}{lll}
\hline values $(\mathrm{ng} / \mathrm{mL})$ & $\mathrm{CVs}(\%)$ & $\mathrm{CVs}(\%)$ \\
& same batch & different batches \\
10 & 9.62 & 13.05 \\
300 & 7.14 & 9.43 \\
\hline
\end{tabular}

\section{Specificity}

\section{Specificity}

Take the prepared standard product thrombopoietin (TPO) and the corresponding standard product Tg and test paper at the same time. The cross-reaction rate $(\mathrm{CR} \%)$ at each concentration was calculate as $\mathrm{TPO} / \mathrm{Tg}$ $\times 100 \%$. Within the concentration range used in this study, the cross-reaction rate with TPO was less than $0.1 \%$, indicating that there was no cross-reaction.

\section{Minimum detection limit}

The $60 \mu \mathrm{L}$ standard blank points were added onto the test strip. After reaction at 30 for 5 min, the test was 
repeated 10 times. The $\mathrm{T} / \mathrm{C}$ ratio was calculated and the concentration was measured using the standard curve. The mean value of $\mathrm{T} / \mathrm{C}$ was 0.062 , which was converted to $0.02 \mathrm{ng} / \mathrm{mL}$ on the standard curve. This value was the sensitivity of the test group.( Table3. Minimum detection limit)

Table3. Minimum detection limit

\begin{tabular}{llll}
\hline No. & C Line & T Line & signal value \\
\hline 1 & 52941 & 4235 & 0.08 \\
2 & 54502 & 2725 & 0.05 \\
3 & 49881 & 3492 & 0.07 \\
4 & 51092 & 2555 & 0.05 \\
5 & 48997 & 2940 & 0.06 \\
6 & 51436 & 2057 & 0.04 \\
7 & 52377 & 2619 & 0.05 \\
8 & 49450 & 3462 & 0.07 \\
9 & 48887 & 4889 & 0.1 \\
10 & 52996 & 2650 & 0.05 \\
$? \mathrm{X}$ & 51255.9 & 3162.276 & 0.062 \\
\hline
\end{tabular}

\section{Tissue test results}

The samples were tissue samples from the pathology department with definite pathological results. Take 14 cases of pathological results for $\mathrm{Tg}$ can normal secretion of thyroid tissue of thyroid carcinoma and 10 cases of pathological results have metastatic lymph node tissue as positive group, 45 cases of pathology confirmed as unable to secrete $\mathrm{Tg}$ of thyroid tissue, including 13 cases of fat tissue, muscle tissue in 7 cases, 10 cases of fibrous tissue, lymph node tissue 15 cases (not shift) as a negative control group separately puncture test. The value of $>35 \mathrm{ng} / \mathrm{mL}$ was positive, the detection rate was calculated, and the concentration was calculated with the 3.1 standard curve. SPSS 19.0 software was used to analyze the concentration values. The paired t-test was used to test the concentration values. $\mathrm{P}<0.05$ was considered to be statistically significant. The results were as follows(Table4. Tissue test results)

Table4.Tissue test results

\begin{tabular}{llllllllll}
\hline Tissue & $1-2$ & $1-3$ & $1-4$ & $1-5$ & $1-6$ & $2-3$ & $2-4$ & $2-5$ & $2-6$ \\
\hline $\mathrm{t}$ & 1.08 & 4.94 & 3.69 & 3.99 & 5.03 & 7.27 & 6.57 & 7.27 & 7.27 \\
$\mathrm{P}$ & 0.31 & 0.00 & 0.01 & 0.00 & 0.00 & 0.00 & 0.00 & 0.00 & \\
\hline
\end{tabular}

1.Thyroid; 2.Lymph(with thyroid cancer cells); 3.Fat; 4. Muscle; 5. Fiber; 6.Lymph(normal); 7.positive group; 8.negative control group

Thyroid tissue and metastatic lymph nodes of tissue results difference $(\mathrm{P}>0.05)$, and other organizations results than there were significant differences $(\mathrm{P}<0.05)$;Had metastatic lymph nodes and other non-thyroid tissue results have significant differences $(\mathrm{P}<0.05)$, results are obvious different positive group and negative control group $(\mathrm{P}<0.05)$. ( Table5.The coincidence rate with pathological results)

Table5.The coincidence rate with pathological results

\begin{tabular}{llll}
\hline pathological results & pathological results & FNA-Tg & FNA-Tg \\
\hline \multirow{3}{*}{ positive group } & Thyroid & - & + \\
& Lymph(lesion) & 0 & 14 \\
& & 0 & 10
\end{tabular}




\begin{tabular}{llll}
\hline pathological results & pathological results & FNA-Tg & FNA-Tg \\
\hline negative control group & Fat & 13 & 0 \\
& Muscle & 7 & 0 \\
& Fiber & 10 & 0 \\
& Lymph(normal) & 15 & 0 \\
\hline
\end{tabular}

The coincidence rate of positive thyroid tissue and negative non-thyroid tissue was $100 \%$ using the Tg-POCT test reagent. Tg-POCT test reagent can be used to distinguish tissues that can normally secrete $\mathrm{Tg}$ from those that cannot.

Discussion

Thyroid cancer is a common endocrine malignant tumor, accounting for approximately $1 \%$ of all malignant tumors [4]. The incidence of thyroid cancer in the Chinese population is $1 / 300,000-1 / 200,000$, with an annual increase of nearly 5 times since the last 10 years. More than $85 \%$ of thyroid cancers are thyroid papillary carcinomas[5]. Lymph node metastasis can occur in the early stage of PTC, but patients can still survive in the long term, provided that treatment is prompt and accurate. For patients with cervical lymph node metastasis, total thyroidectomy and regional lymph node dissection are generally required, after which thyroxine is administered, and $\mathrm{I}^{131}$ nuclide ablation is required for high-risk patients. Generally, 5\%-20\% in situ or local recurrence and 10\%-15\% distal metastasis will occur after surgery[6-8]. Therefore, accurate preoperative identification of the nature of thyroid mass and cervical lymph nodes is crucial for the selection of reasonable surgical methods and treatment programs; in addition, rapid intraoperative identification of the nature of cervical metastatic lymph nodes is particularly important for favorable prognosis of thyroid tumor patients.

At present, the diagnostic methods for suspected cervical metastatic lymph nodes of thyroid cancer mainly include high-frequency ultrasound (B ultrasound), neck CT, fine needle aspiration biopsy cytology (FNAC), and other examination methods. Among them, b-mode ultrasound is a convenient and harmless examination method, which can assist in the identification of benign and malignant thyroid nodules, but its main disadvantages are that it cannot reflect the histological characteristics of nodules and that b-mode ultrasound examination is relies heavily on the experience of the examiner, thus being subjective to some extent. The diagnostic value of neck $\mathrm{CT}$ for thyroid nodule lesions is not well reported in the literature and is rarely used in the clinical setting. B-ultrasound guided FNAC is currently recognized as the most direct method to identify the nature of the mass and lymph node. It has been reported that the sensitivity and specificity can reach $87 \%$ and $76 \%$, respectively, but $20 \%-40 \%$ of puncture specimens cannot be accurately identified, and cytological examination results are quite influenced by the experience of the technician[9-10]. The above diagnostic methods are mainly used for preoperative identification and prognosis, but these cannot be used intraoperatively due to the need for large instruments or the prolonged examination time.

Traditional methods for Tg detection mainly include radioimmunity, enzyme-linked immunoassay, and chemiluminescent immunoassay, but these methods require large instruments and require a long detection time, which are not suitable for rapid intraoperative detection. Fluorescence microsphere immunochromatography is a novel, innovative quantitative detection technology developed by combining time-resolved fluorescence immunoassay and traditional immunochromatography. It has the characteristics of high sensitivity, good stability [11], and short detection time.

\section{Conclusions}

In this study, a rapid intraoperative detection method for Tg was developed, which is characterized by good stability, high sensitivity, and high specificity. Combined with intraoperative fine needle puncture, it can quickly help to detect thyroid cancer metastasis in the lymph nodes, which will help to improve the efficiency and quality of surgical treatment.

Conflicts of interest 
There are no conflicts to declare.

Acknowledgements

This work was supported by the Innovation Capacity Development Plan of Jiangsu Province (BM2018023-2), Jiangsu Provincial Key Medical Discipline (Laboratory)(ZDXKA2016017), the Technology Research Program of Shiyan City(19Y86), Medical and Public Health Project of Wuxi Sci-Tech Development Fund (No WX18IIAN047) and AD7C-NTP in alzheimer's disease of Wuxi area(MS201803).

Notes and references

[1] Chisholm EJ,Economides P,Elmiyeh B,et al.Pictorialultrasoundreports combined with fine needle washout thyroglobulin assay to aid thyroid surgery[J].Laryngoscope,2011,121(6):1231-1232.

[2] Bruno R,Giannasio P,Chiarella R,et al. Identification of a neck lump as a lymph node metastasis from an occult contralateral papillary microcarcinoma of the thyroid:key role of thyroglobulin assay in the fine-needle aspirate[J]. Thyroid,2009,19(5):531-533.

[3] Eichhom W, Tabler H Lippold R, etal.Prognoslic factors detrermining long-term survival in well-differentiated thyroid cancer;an analysis of four hundred eighty-four patients undergoing therapy and aftercare at the same institutionl[J].Thyroid,2003,13(10):949-958.

[4]Ye ZQ, Gu DN,Hu HY, et al. Hashimoto's thyroiditis, microcalfication andraised throtropin levels within normal range are associated with thyroid cancer [J].World J Surg Oncol, 2013, 11:56 and follicularthyroid carcinoma. Thyroid,1999,9(5):421-427.

[5] Zhou hao, Chen haizhen, Chen xi, Yu ningkang, etal. Determination of thyroid globulin in fine needle eluent to identify the nature of metastatic lymph nodes. Surgical theory and practice, 2012, 17(1):17-20

[6] Uruno T,Miyauchi A,Shimizu K,et al. Usefidness of thyreglobulin measurement in fine-nellie aspiration biopsy specimens for diagnosing cervical lymph node metastasis in patients with papillary thyroid cancer.World J Surg,2005,29(4):483-485.

[7] Schlumberger MJ. Papillary and follicular thyroid eareinom. NEngl J Med,1998,338(5):297-306.

[8] Mazzaferri E L.An overviow of the management of papillary

[9] Gao xuwen, Li jilian. Thyroid disease. People's medical publishing house, 2000:178-180.

[10] Ogilvie JB.Piatigonsky EJ,Clark OH.Current status of fine needle aspiration for thyroid nodules[J].Adv Surg,2006,40(6):223-238.

[11] Wang diqiang, Liu baoling, Hu jie, etal. Preparation and application of fluorescent microspheres. Polymer materials science and engineering, 2004, 20(4):42-45

Figure1. Labeling of fluorescent microspheres

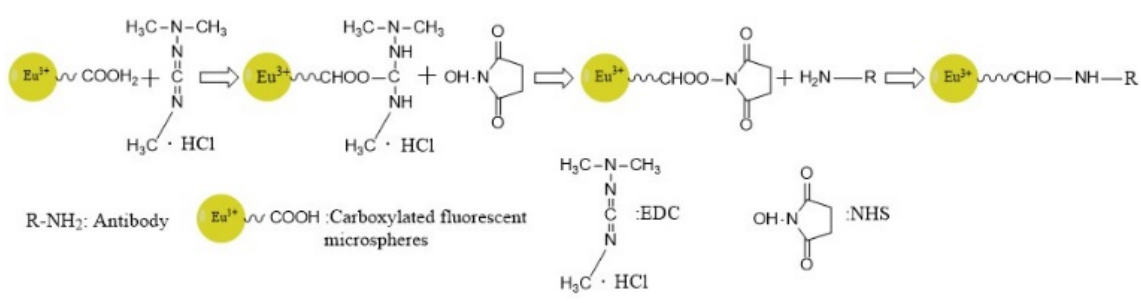

Figure2. The composition of speed measuring card 


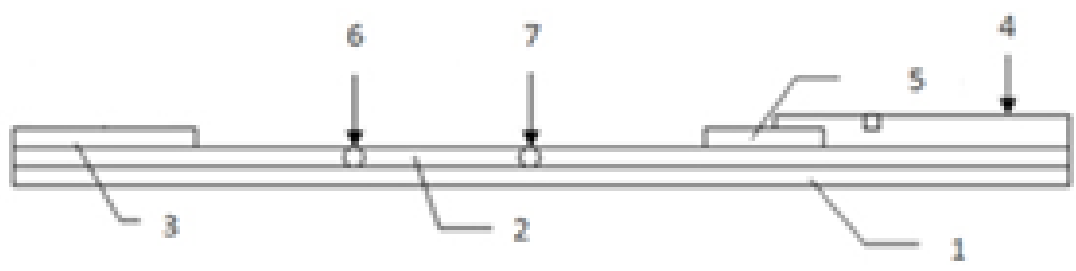

1.plastic back plate 2 .analyzing membrane 3 .water absorbing paper 4.sample pad 5.binding pad 6.control line 7 .test line

Figure3. Testing Flow
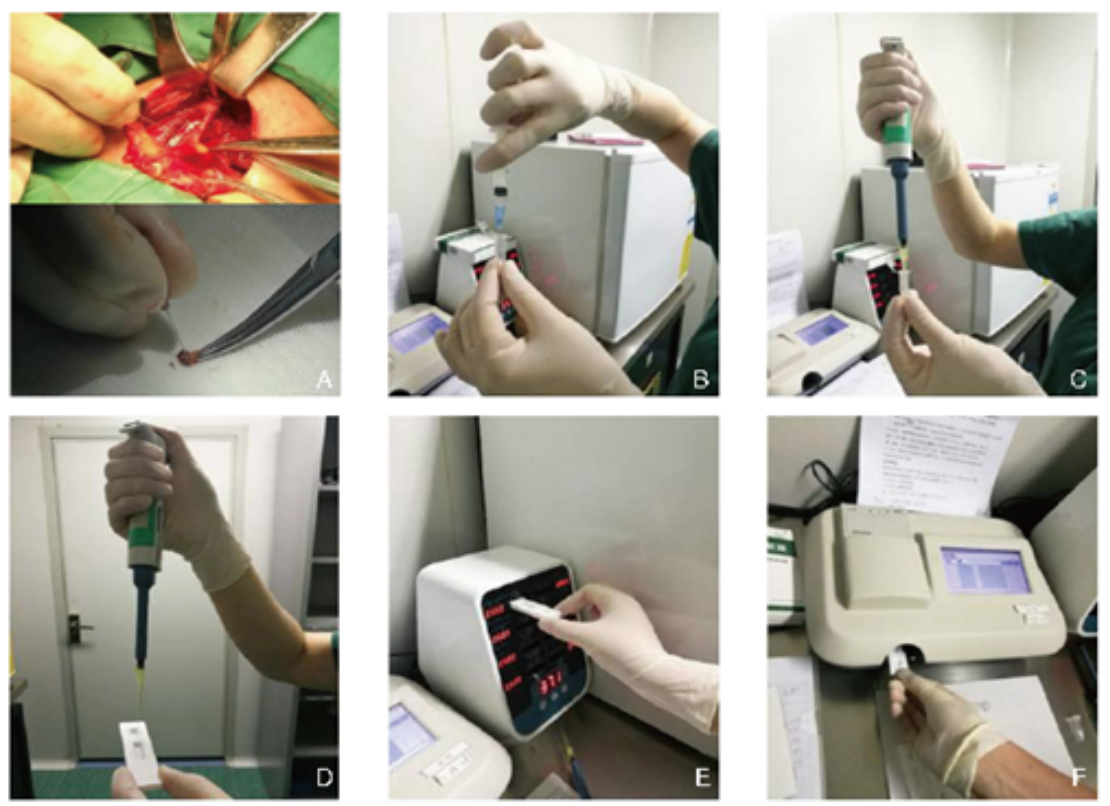

Figure4. effect of $\mathrm{pH}$ on activation 


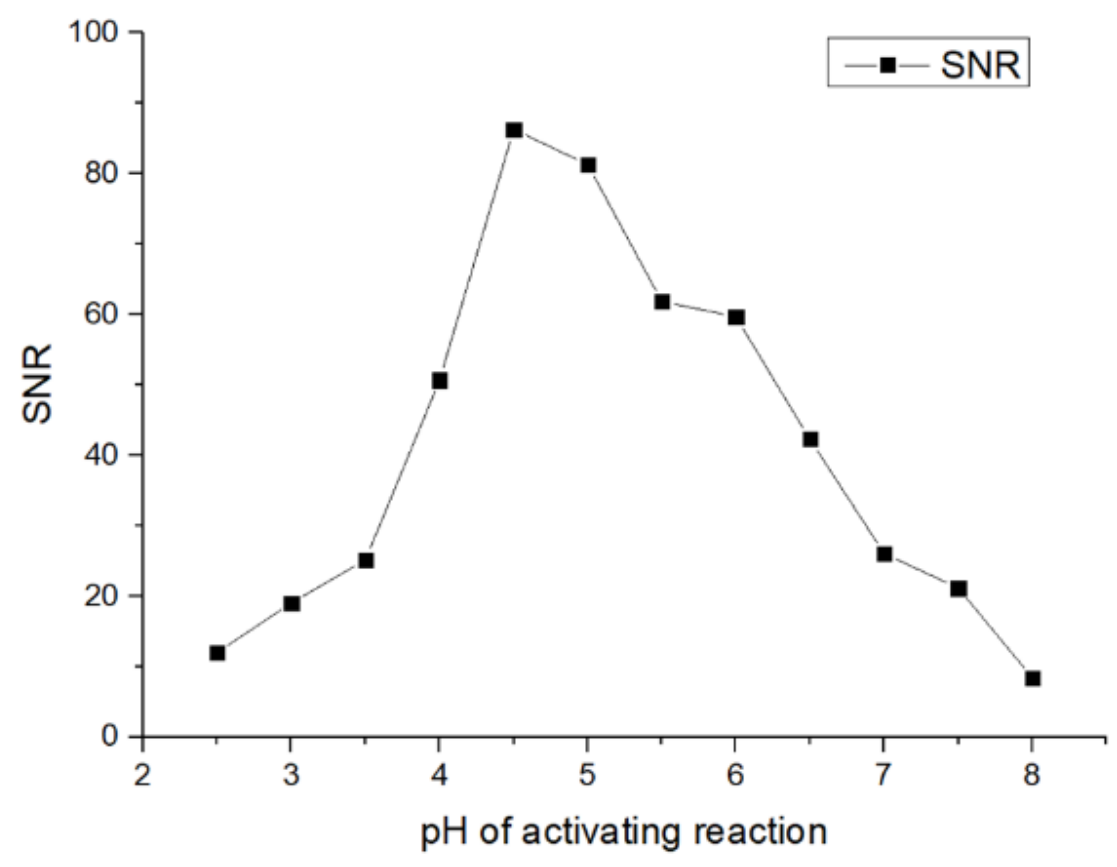

Figure5. effect of $\mathrm{pH}$ on coupling

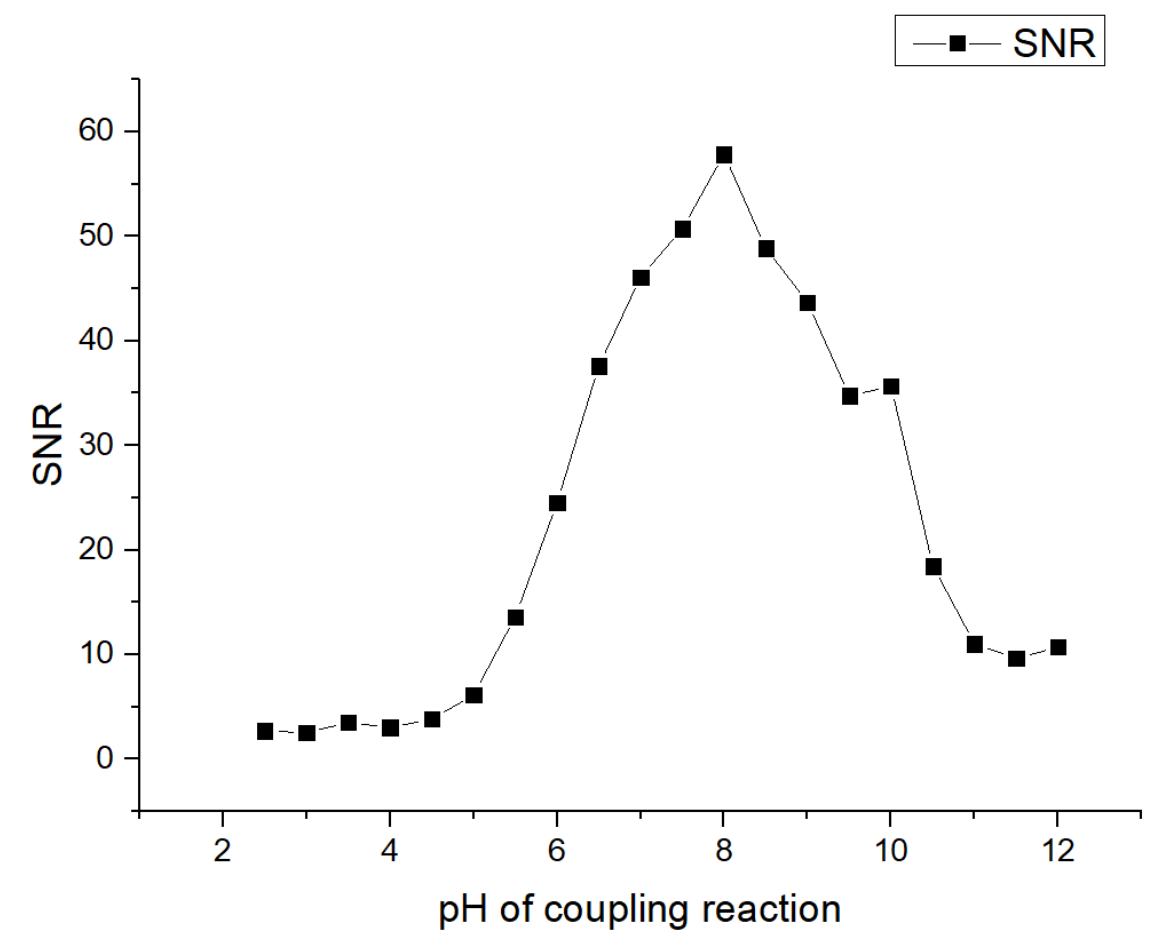

Figure6.effect of buffer solution 


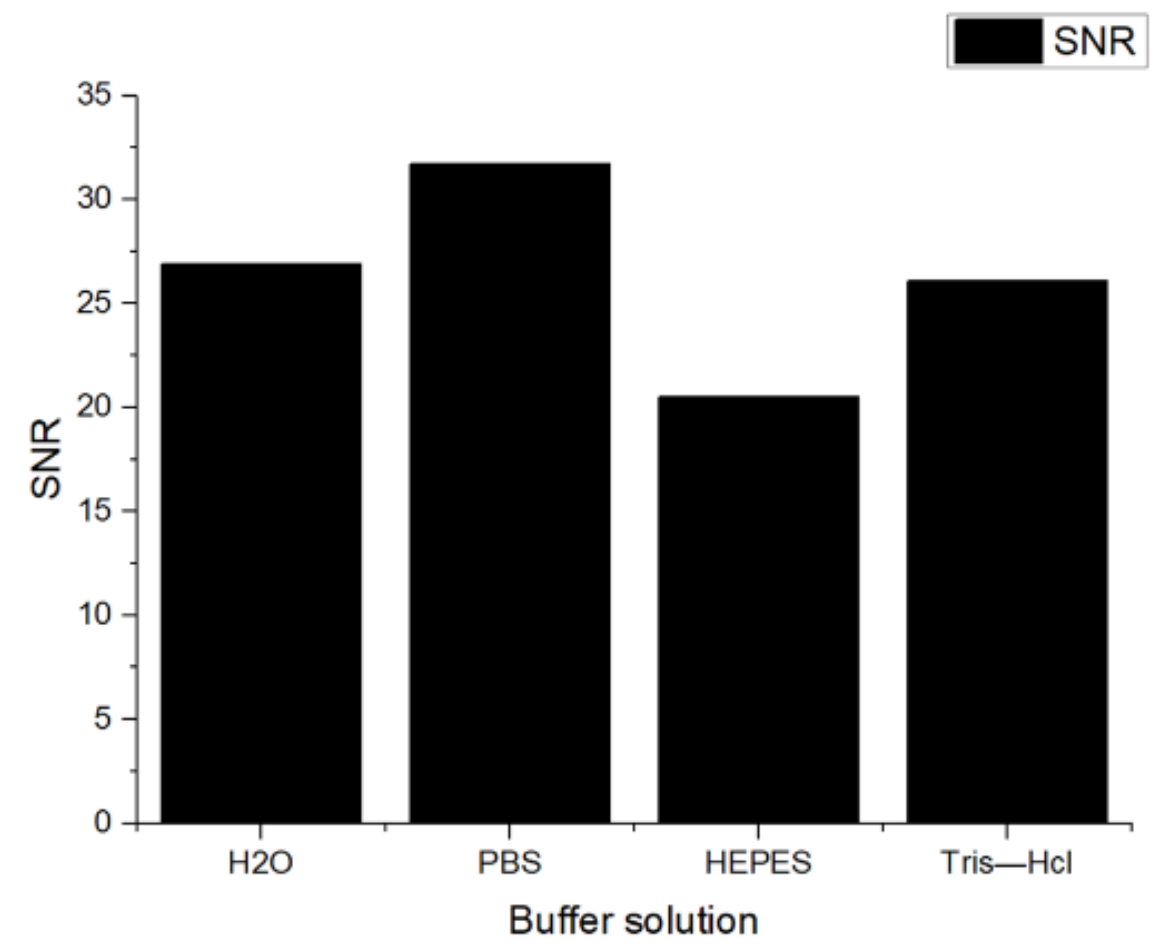

Figure7. effect of antibody labelled amount

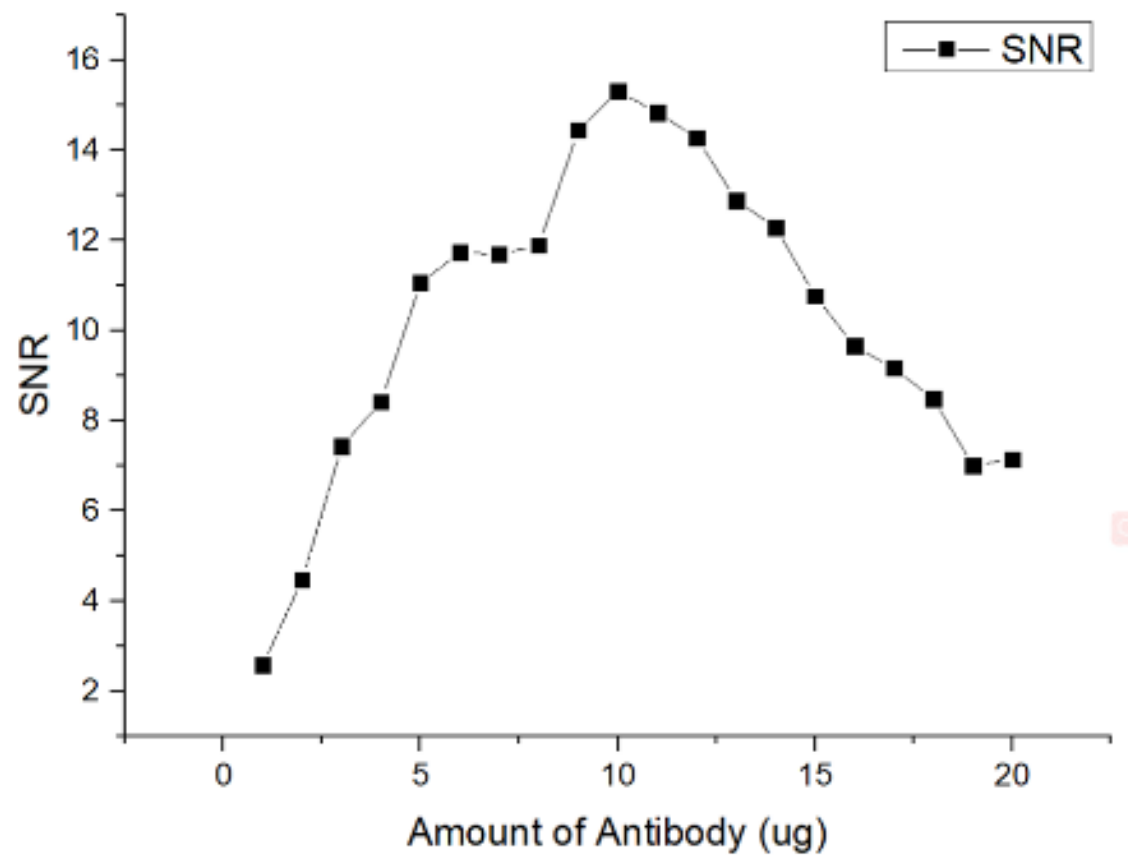

Figure8.effect of antibody coated amount 


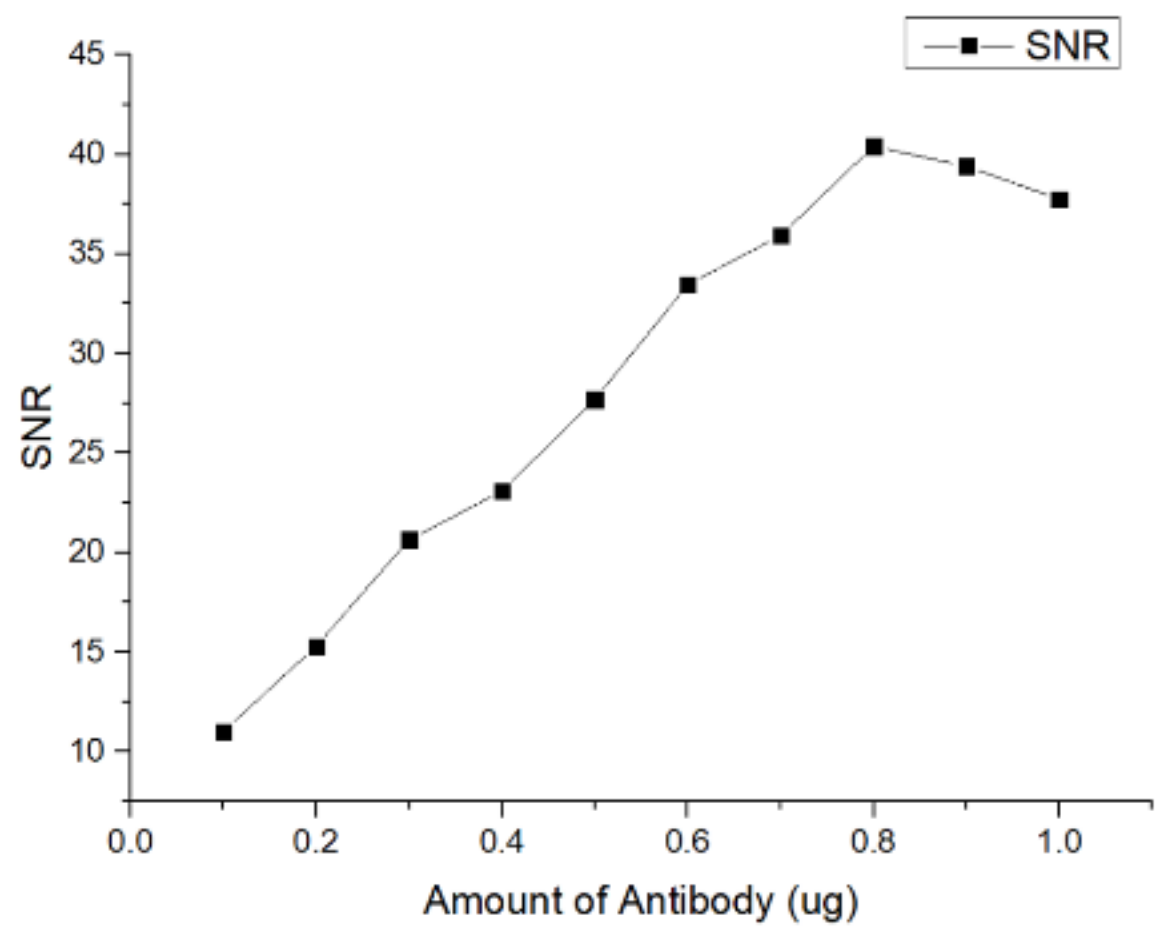

Figure9.effect of dilution ratio of fluorescent microsphere

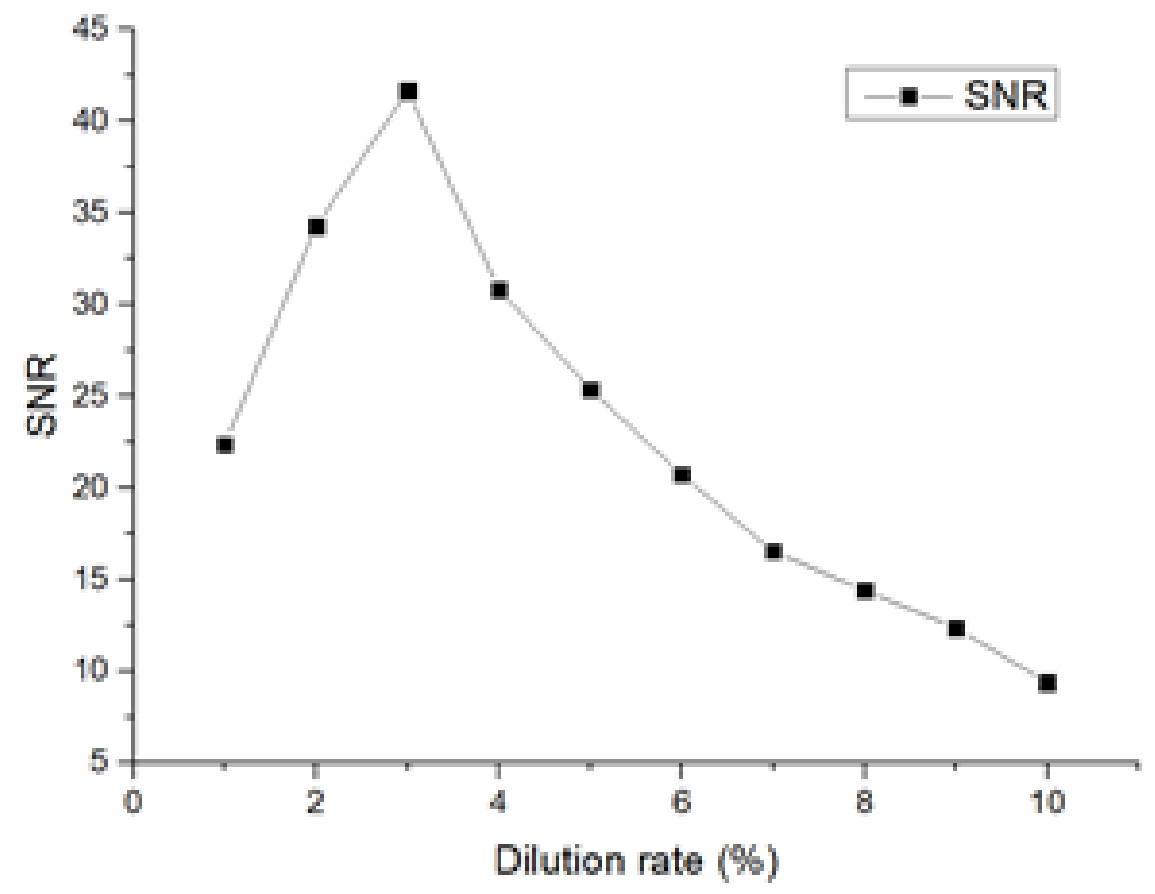


Figure10. standard curve

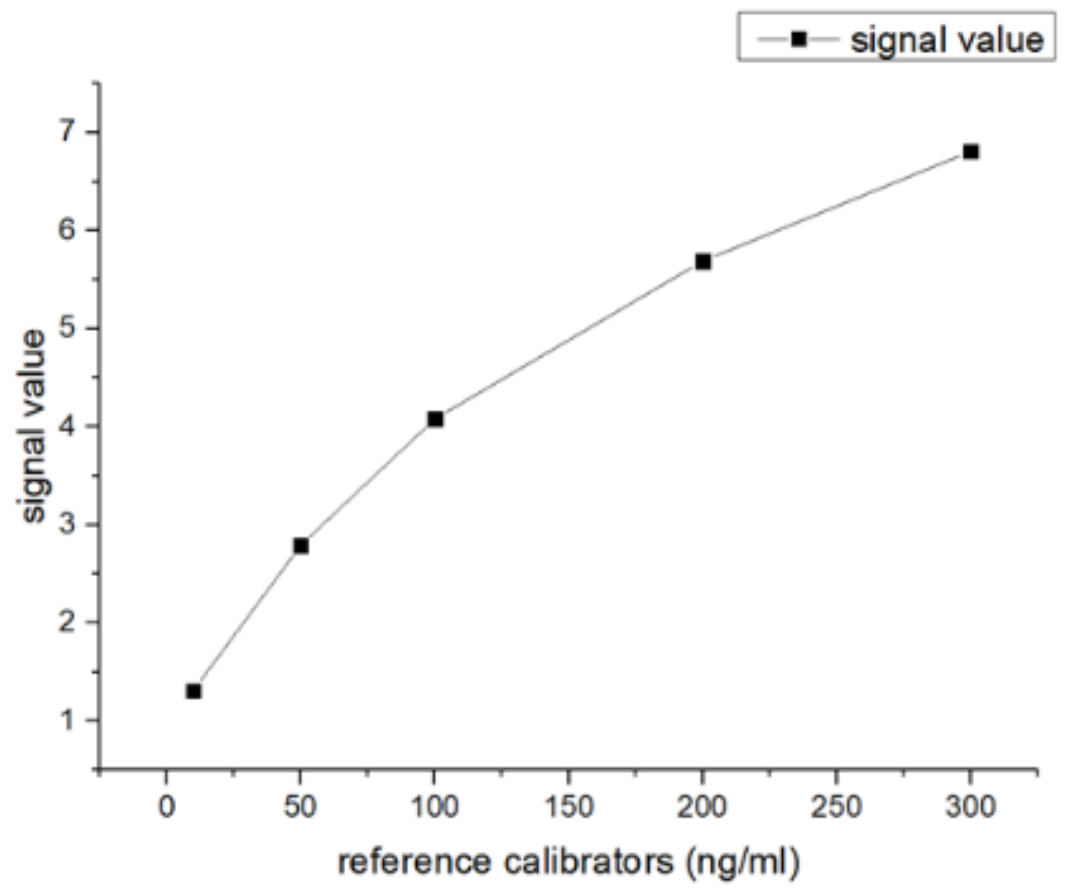

\section{Hosted file}

Rapid-intraoperative-method-for-the-identification-of-metastatic-lymph-nodes-from-thyroid-carcinoma.doc available at https://authorea.com/users/316120/articles/446337-rapid-intraoperative-method-forthe-identification-of-metastatic-lymph-nodes-from-thyroid-carcinoma
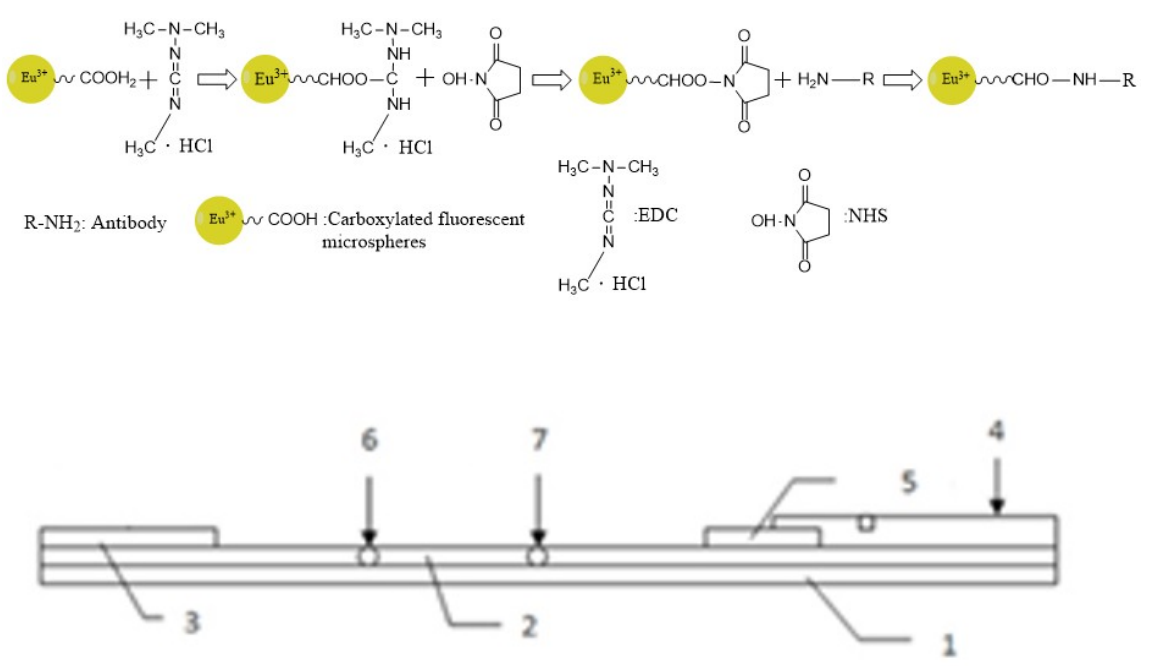

1.plastic back plate 2.analyzing membrane 3 .water absorbing paper 4.sample pad 5.binding pad 6.control line 7.test line 

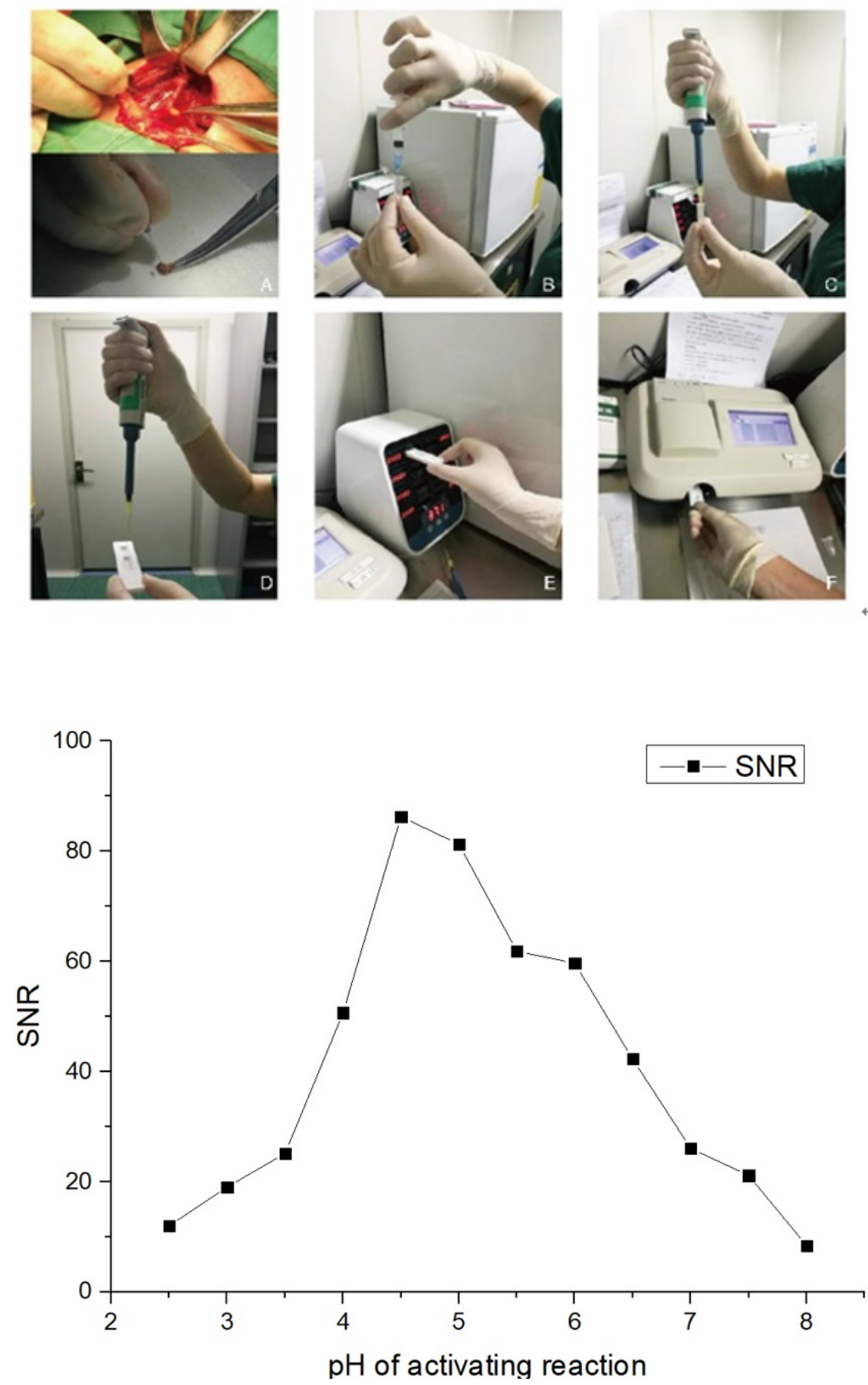

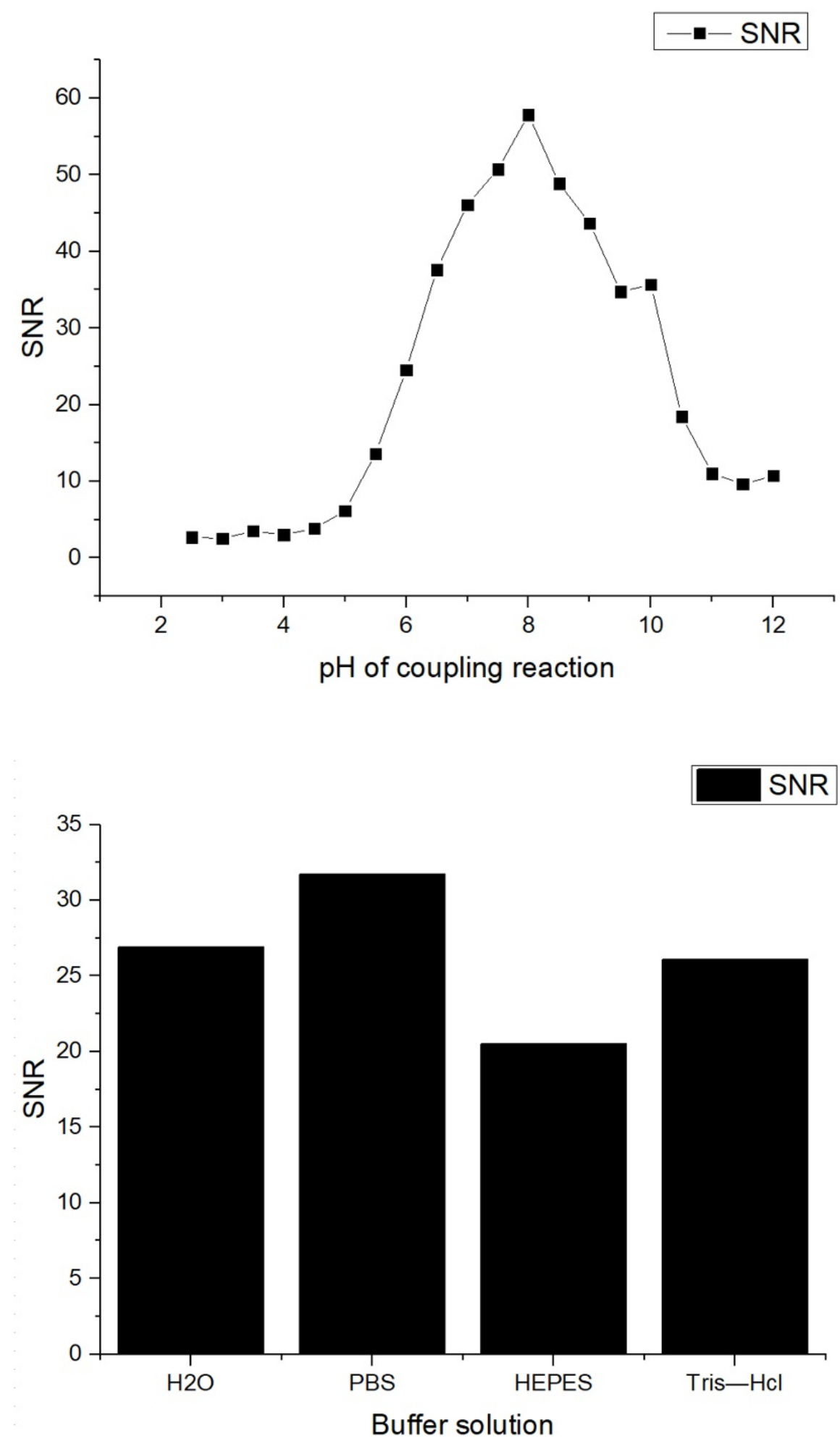

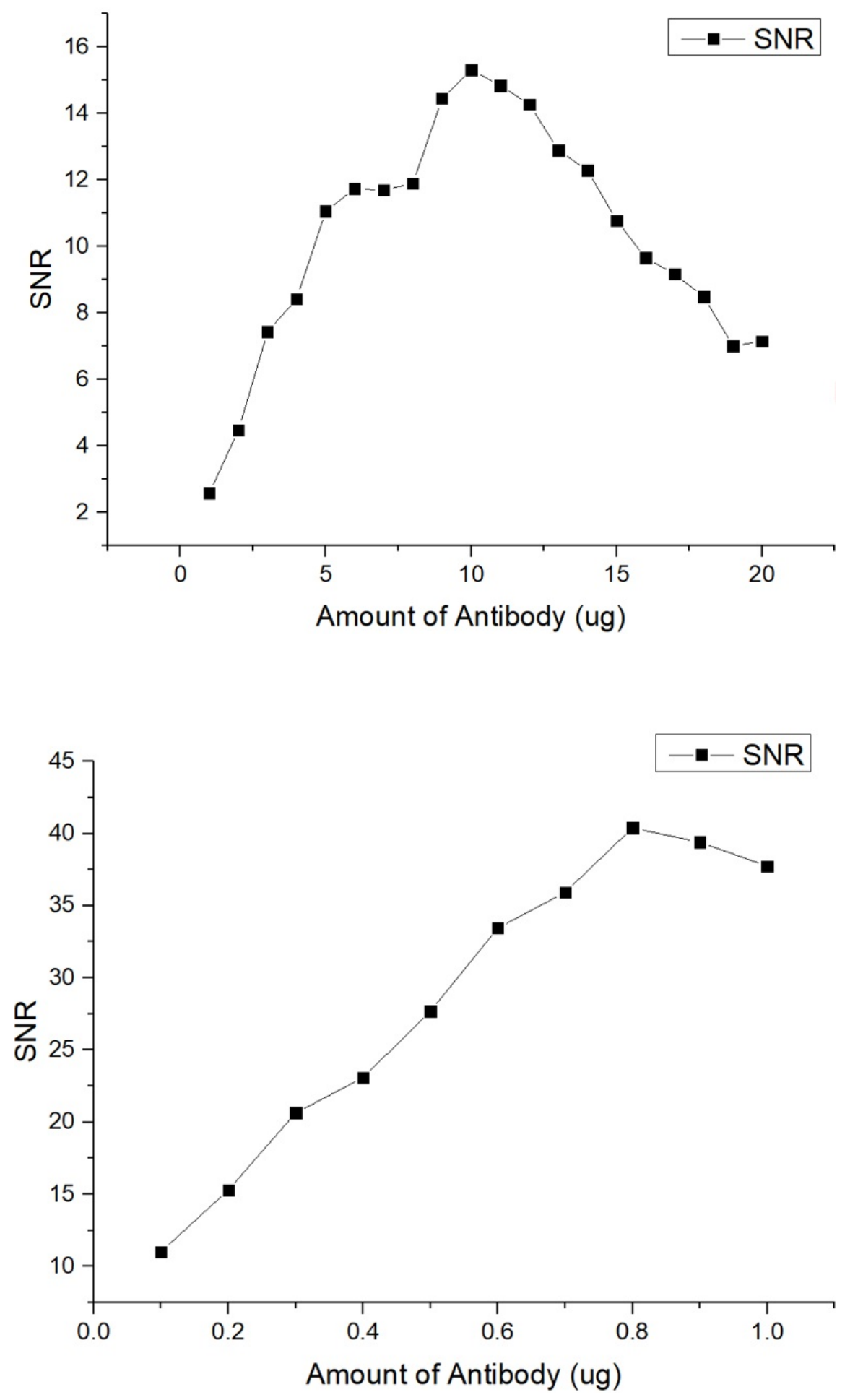

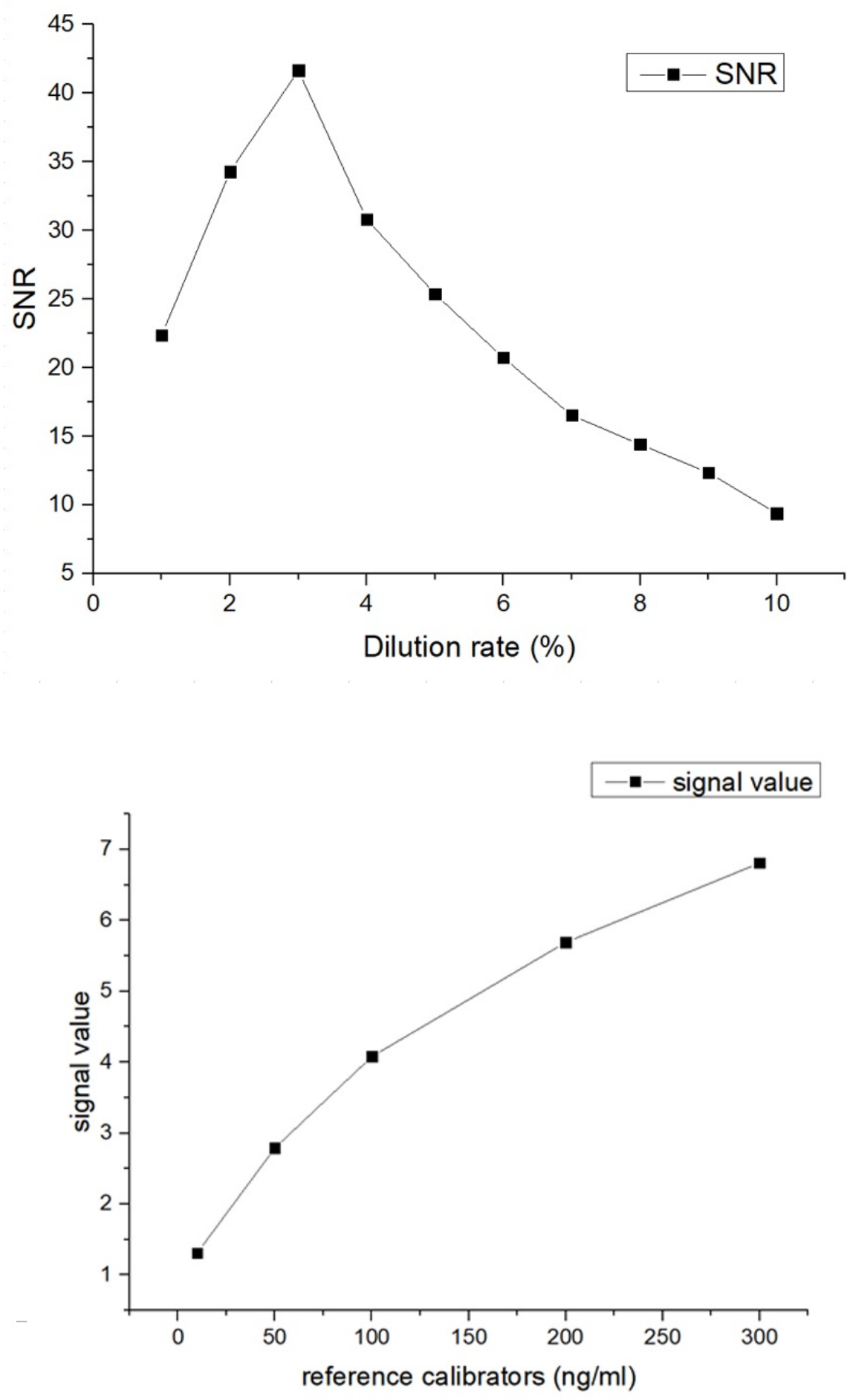